\title{
Henoch-Schönlein purpura nephritis associated with monoclonal gammopathy of renal significance: a case report
}

\author{
Hui Zhao (iD), Wen-hui Huang (iD, Jun-yue Huang (iD, Shou-yan Lu (iD, Ya-hong Yang (i), and \\ Zhi-gang Ma 10 \\ Department of Nephrology, Gansu Provincial Hospital, Lanzhou, Gansu, China
}

\begin{abstract}
Monoclonal gammopathy of renal significance (MGRS) can present with different morphologic features and lead to kidney failure. The Henoch-Schönlein purpura nephritis (HSPN) that cannot be relieved by treatment with glucocorticoid and immunosuppressive agents suggests the presence of monoclonal gammopathy in adult patients. The present study reports on a single case of HSPN associated with IgA-kMGRS. The patient who suffered from recurrent skin purpura for 6 months and nephrotic syndrome for 2 months was admitted to our hospital. Bone marrow biopsy showed monoclonal gammopathy of undetermined significance. Kidney biopsy indicated a Henoch-Schönlein purpura nephritis (HSPN, ISKDC classified as type III) with positive staining with $\kappa$-light chain in the glomeruli and renal tubular epithelial cells. Furthermore, skin biopsy showed leukocytoclastic vasculitis and negative staining for Congo red and light chain. Given both the renal and cutaneous involvement, the patient was considered to have HSPN associated with IgA-kMGRS. The patient experienced an exacerbation in his purpura-like lesions and clinical status after treatment with glucocorticoid and immunosuppressive agents. Consequently, the patient was put on a regimen that included dexamethasone (20 mg on the 1st, 4th, 8th, and 11th days of each month, $i v)$ and bortezomib (2.4 $\mathrm{mg}$ on the 1st, 4th, 8th, and 11th days of each month, iv). Eight weeks after treatment, he had complete resolution of his cutaneous purpura and his biochemical parameters improved. The latent presence of MGRS in cases of HSPN should be considered in adult patients. Increased cognizance and correct treatment options could improve patient outcomes.
\end{abstract}

Key words: Monoclonal gammopathy of renal significance; Henoch-Schönlein purpura nephritis; Chemotherapy

\section{Introduction}

Henoch-Schönlein purpura (HSP) is a systemic vasculitis of small and medium-sized vessels. The clinical presentation of HSP involves cutaneous palpable purpura, arthralgia/arthritis, bowel angina, and hematuria/proteinuria (1). HSP is rarely seen in adults, with an estimated annual incidence of 1.3 cases per 100,000 . The disease tends to be self-limited in children $(2,3)$, while it is more severe in older patients $(3,4)$. Skin purpura, a classical feature of HSP, can be also observed in patients with autoimmune diseases and hematological malignancies, including multiple myeloma and amyloidosis.

Monoclonal gammopathy (MG) is a result of a clonal proliferation of lymphocytes or plasma cells that involves three main components: skin, kidneys, and peripheral nervous system (nerves). MG of renal significance (MGRS) involves the kidney and can lead to kidney failure $(5,6)$. Morbidities associated with MGRS are high due to the severe renal lesions and associated systemic alterations (7). The present study reports a single case of a patient with Henoch-Schönlein purpura nephritis associated with IgA-кMGRS that was gradually improved by dexamethasone and bortezomib chemotherapy.

\section{Case Report}

A 61-year-old male retired teacher who suffered from skin purpura was admitted to the local hospital. The patient was diagnosed with HSP and received oral prednisone (30 mg/day) in July 2016. Approximately one week later, the purpura disappeared, but it recurred after 20 days. Full blood examination appeared normal, as well as serum creatinine, serum calcium, liver function, and serum immunoglobulin (lg) levels. The patient's 24-h urine protein was $0.352 \mathrm{~g} /$ day, which was above the reference range of $0.00-0.14 \mathrm{~g} /$ day $)$. The patient was then prescribed prednisone ( $30 \mathrm{mg} /$ day) combined with angiotensin

Correspondence: Zhi-gang Ma: <fimmu@sohu.com> 
receptor antagonist. Ten days later, his purpura disappeared, and the prednisone dose was gradually reduced to $15 \mathrm{mg}$ daily. While on prednisone, his purpura recurred intermittently and was therefore was admitted to Gansu Provincial Hospital in July 2017 for further testing. He initially declined a renal biopsy, and was prescribed an increased dose of oral prednisone ( $40 \mathrm{mg} /$ day), which was not effective. Two months later, 24-h urine protein increased to $3.399 \mathrm{~g} /$ day. His treatment was altered to a reduced dose of prednisone (15 mg/day) combined with cyclosporin A (100 mg, bid). In October 2017, the proteinuria improved (24-h urine protein decreased to $0.828 \mathrm{~g} /$ day), but he developed a purpuric rash on his extremities and torso. Consequently, he was hospitalized with purpuric skin lesions and edema on his lower limbs.

Urine analysis showed microscopic hematuria; serum albumin was decreased to $37.5 \mathrm{~g} / \mathrm{L}$ (reference range 40-55 g/L) with an otherwise normal liver. Erythrocyte sedimentation rate was elevated $(29 \mathrm{~mm} / \mathrm{h}$, reference range $0-15 \mathrm{~mm} / \mathrm{h}$ ). Serological tests for hepatitis $B$, hepatitis $\mathrm{C}$, and human immunodeficiency virus, as well as cryoglobulin, cold agglutinin, and C3 and C4 were all negative. Serum immunoglobulin (lg) levels showed elevated $\lg A(6.35 \mathrm{~g} / \mathrm{L}$, reference range $0.82-4.53 \mathrm{~g} / \mathrm{L})$, but decreased $\operatorname{lgG}(6.44 \mathrm{~g} / \mathrm{L}$, reference range $7.51-15.6 \mathrm{~g} / \mathrm{L})$ and $\operatorname{lgM}(0.40 \mathrm{~g} / \mathrm{L}$, reference range $0.46-3.04 \mathrm{~g} / \mathrm{L})$. Serum free light chains showed normal $\kappa$ light chain levels (1.77 g/L, reference range 1.70-3.70 g/L) and decreased $\lambda$ light chain $(0.71 \mathrm{~g} / \mathrm{L}$, reference range 0.90 $2.10 \mathrm{~g} / \mathrm{L}$ ), with a normal $\kappa / \lambda$ ratio of 2.49 (reference range 1.47-2.95). Serum immunofixation electrophoresis pattern showed that there was an $M$ protein band on the electrophoretic pattern (ELP), which formed a specific reaction precipitation zone with anti-lgA and anti- $\kappa$ chain. In addition, urine light chain analysis revealed Bence-Jones proteinuria with $\lambda$ chain level of $0.04 \mathrm{~g} / \mathrm{L}$ (reference range $0 \mathrm{~g} / \mathrm{L}$ ) and $\kappa$ chain level of $0.11 \mathrm{~g} / \mathrm{L}$ (reference range $0 \mathrm{~g} / \mathrm{L}$ ). The graph of urine Bence-Jones protein electrophoresis also showed that there was an M protein band on the ELP. Investigations for autoimmune and infective causes were negative. Echocardiography and X-ray of the chest, skull, and pelvis were normal. Bone marrow biopsy revealed increased plasma cells accounting for $0.67 \%$ of the marrow cellularity.

\section{Kidney biopsy}

The patient underwent a kidney biopsy in November 2017 that revealed HSP nephritis (HSPN, ISKD classified as type III, defined as focal hyperplasia and sclerosis), with isolated positive $\kappa$-light chain and negative lambda light chain immunostaining (Figure 1). Light microscopy indicated a proliferation of mesangial cells and mesangial matrix, with only a minority of tissue samples showing segmental sclerosis. Segments of some glomeruli showed the mesangial matrix expanding into the endothelial cells. Congo red staining for amyloid and IgG subclass was negative. Moderate confluent granular staining of $\lg \mathrm{A}$ deposits was identified in the mesangium. Renal interstitial fibrosis was $<10 \%$. Electron microscopy demonstrated electron-dense deposits in the mesangial region.

\section{Skin biopsy}

In November 2017, the patient underwent skin biopsy that showed leukocytoclastic vasculitis and negative staining for Congo red and $\kappa$-light chain (Figure 2).

\section{Clinical follow-up}

The patient received intravenous methylprednisolone (320 mg/day) for seven days, and intravenous cyclophosphamide (600 mg/week) combined with intravenous dexamethasone $(10 \mathrm{mg} /$ week) for the next three weeks. Nevertheless, no improvement in purpura was observed one month after therapy. In addition, his serum creatinine levels increased to $2.85 \mathrm{mg} / \mathrm{dL}$ (reference range, 0.59 $1.20 \mathrm{mg} / \mathrm{dL}$ ); hemoglobin decreased to $62 \mathrm{mg} / \mathrm{dL}$ (reference range, 120-160 mg/dL), and proteinuria (24-h urine protein, $5.4 \mathrm{~g} /$ day) did not improve. One month later, the patient presented with worsened edema and lethargy. His serum creatinine further increased to $3.08 \mathrm{mg} / \mathrm{dL}$ while his serum albumin decreased to $22.7 \mathrm{~g} / \mathrm{L}$. Considering that his condition continued to deteriorate, the patient was placed on our recommended regimen that included dexamethasone $(20 \mathrm{mg}$ on the $1 \mathrm{st}, 4 \mathrm{th}, 8 \mathrm{th}$, and 11th days of each month, iv) and bortezomib (2.4 mg on the 1st, 4th, 8th, and 11th days of each month, iv). Eight weeks after treatment, the patient had complete resolution of the cutaneous purpura, edema, and lethargy. His biochemical parameters also improved, and his serum creatinine decreased to $1.00 \mathrm{mg} / \mathrm{dL}$, serum albumin increased to $39.9 \mathrm{~g} / \mathrm{L}$, and 24-h urine protein decreased to $1.9 \mathrm{~g} / 24 \mathrm{~h}$. His serum $\kappa$ light chain and IgA level returned to normal. Following six courses of chemotherapy, the skin lesions were markedly improved. In August 2018, follow-up examination showed that the serum $\mathrm{k}$ light chain and the serum creatinine were normal, serum albumin increased to $42.1 \mathrm{~g} / \mathrm{L}$, hemoglobin increased to $110 \mathrm{mg} / \mathrm{dL}$, and 24-h urine protein decreased to $0.168 \mathrm{~g} / \mathrm{day}$. He has had no further relapses since starting this regime and there has been no further significant proteinuria or hematuria.

\section{Diagnosis}

Based on the above results, the patient was diagnosed with HSPN associated with $\lg A-\kappa$ MGRS.

\section{Discussion}

In children, HSP is usually self-limiting, while severe cases are more commonly seen in adults, and it usually responds to steroids (3). An intriguing aspect of this case involved the potential relationship between the patient's refractory HSP and a hematological malignancy. Recurrent purpura and nephritis may occur in paraproteinemias (8). 

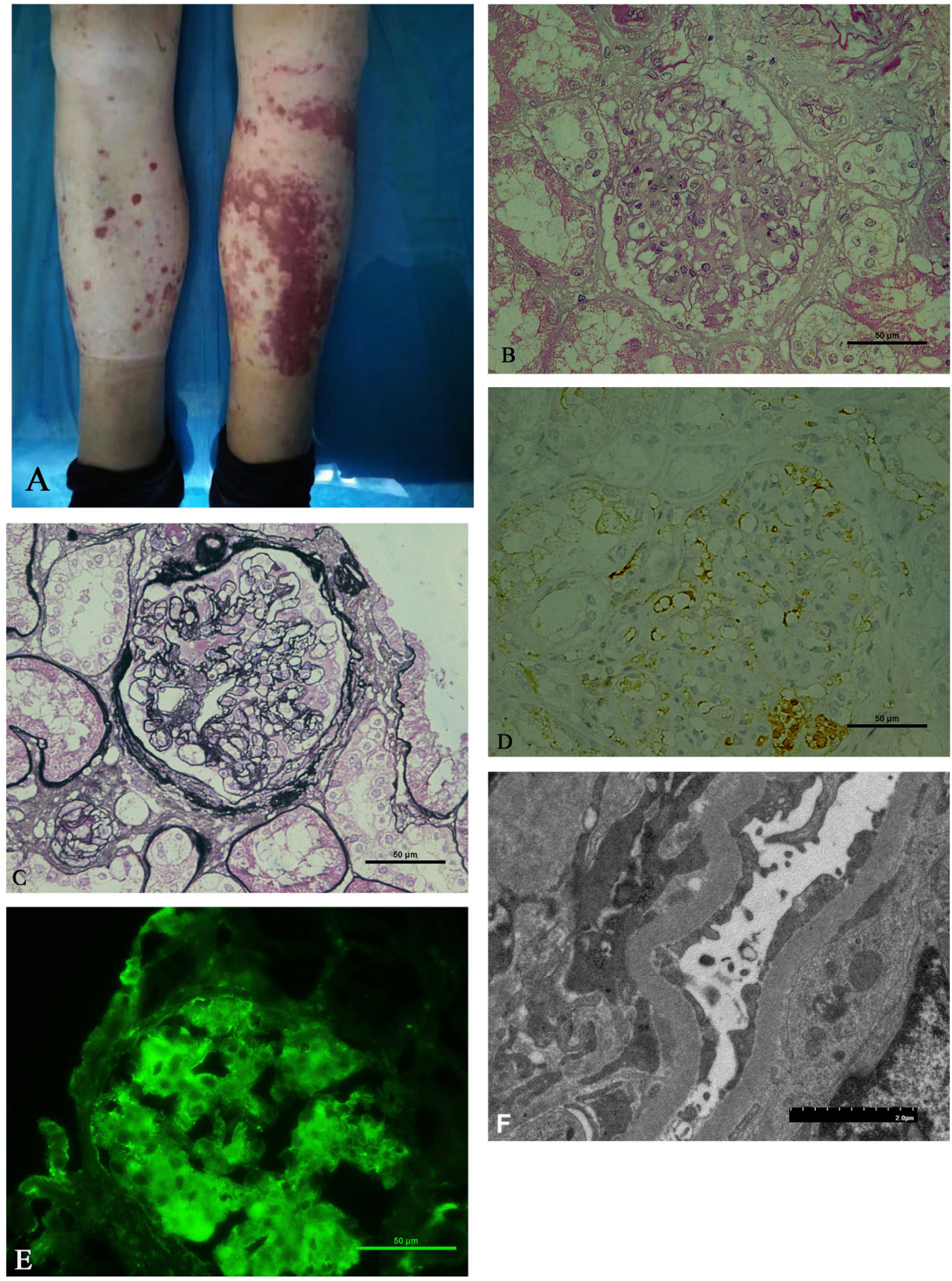

Figure 1. Skin purpura and kidney biopsy. A, Lower extremity purpura. B, Hematoxylin and eosin stain showing glomerular necrosis $(\times 400)$. C, Periodic Schiff-Methenamine stain showing proliferation of mesangial cell and mesangial matrix $(\times 400)$. D, Positive staining with $\kappa$-light chain in the glomeruli and renal tubular epithelial cells $(\times 400)$. E, Confluent, granular staining of mesangial IgA deposits $(\times 400)$. F, Electron microscopy demonstrating the electron-dense deposits in the mesangial region $(\times 5000)$. Scale bars in B, C, D, E: $50 \mu \mathrm{m}$; scale bar in F: $2 \mu \mathrm{m}$.

Skin purpura can also occur in hematological malignancies such as multiple myeloma (MM). Cutaneous involvement associated with MM varies from 5 to $10 \%$ (9). Our patient did not meet the diagnostic criteria for MM. In addition, cryoglobulinemia can induce a thrombotic vasculopathy, and the first clinical lesion is usually a stellar or retiform purpura, which evolves into necrosis. It initially occurs on cold-exposed areas such as helix, tip of the nose, fingers, 

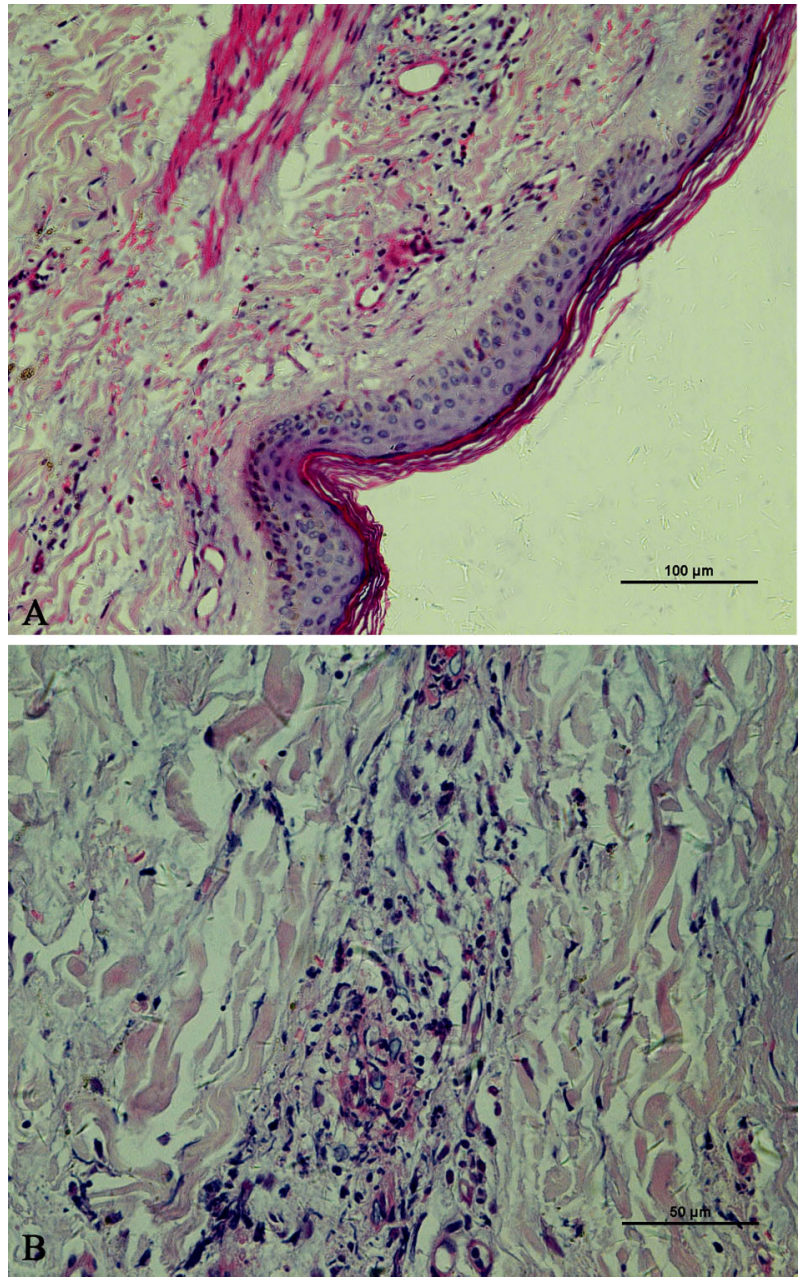

Figure 2. Hematoxylin and eosin stain showing chronic inflammatory cell infiltration around small blood vessels $(\mathbf{A}, \times 200)$ and fibrous connective tissue hyperplasia $(B, \times 400)$. Scale bars: $100 \mu \mathrm{m}(\mathbf{A})$ and $50 \mu \mathrm{m}(\mathbf{B})$.

and toes (10). Our patient's cryoglobulin was negative. However, the skin biopsy revealed leukocytoclastic vasculitis and negative staining for Congo red and $\kappa$-light chain, without $\lg A$ deposition. There was no direct evidence of skin purpura-like lesions with monoclonal gammopathy and IgA vasculitis. Purpuric skin lesions in our patient spread to his extremities 1 year before the current presentation. Peculiarly, the patient experienced an improvement in skin purpura and proteinuria at the beginning of treatment with glucocorticoid, followed by an exacerbation in his purpura-like lesions and clinical status after treatment with glucocorticoid and immunosuppressive agents. Given the clinical severity, the patient was treated with dexamethasone and bortezomib. After eight weeks of treatment, he had a complete resolution of his cutaneous purpura and biochemical parameters. Therefore, HSPN associated with $\lg A-\kappa$ MGRS was thought to be responsible for purpuric skin lesions in this patient. Accordingly, our patient was diagnosed with HSPN with IgA-кMGRS upon hospitalization.

In 2012, the term MGRS was introduced to distinguish monoclonal gammopathies that result in the development of kidney disease from those that are benign. MGRS patients have a small B-cell clone and a low level of circulating paraprotein (11). In April 2017, the International Kidney and Monoclonal Gammopathy Research Group (IKMG) redefined MGRS as a clonal proliferative disorder that produces a nephrotoxic monoclonal immunoglobulin and does not meet previously defined hematological criteria for treatment of a specific malignancy (12). Renal damage is caused by the deposition of secreted monoclonal immunoglobulin (Mlg) or its fragments, produced by a B-cell or plasma cell clone. Kidney biopsy is therefore required to determine the exact nature of the lesion and severity of renal disease, and immunofluorescence and electron microscopy studies are often required to determine the clonality and the deposit pattern (13).

In MGRS cases, tumor burden is not high and the determination of the latent renal injury is based on the physicochemical property of the paraprotein (5). Monoclonal immunoglobulin-related diseases tend to be progressive and are unlikely to undergo spontaneous remission (14,15). MGRS is associated with high morbidity, and treatment is usually necessary to prevent deterioration of renal function. In most cases, overall survival of patients with MGRS is significantly better than that of patients with multiple myeloma, but the renal outcomes are not as good (16). In the most recent observational studies, nine out of 2935 monoclonal gammopathy of undetermined significance (MGUS) patients (approximately $1.5 \%$ ) were diagnosed with MGRS. Among MGRS patients, the incidence of progression to MM was significantly higher than in MGUS patients (18 vs 3\%) (17).

To date, there is no available strategy that could inhibit or clear the monoclonal immunoglobulin tissue deposition. Monoclonal diseases are poorly responsive to conventional immunosuppression and instead require clonedirected therapy (18). Therefore, targeting the underlying B-cell clone with chemotherapy, although it is not an evidently malignant clone per se, is the only available therapeutic option for MGRS (19). The choice of chemotherapeutic agents should take into account the drugs' renal clearance and potential renal and non-renal toxicity (20). The introduction of novel agents like bortezomib has changed the course of myeloma patients on the overall and renal survival curves (21). The Greek myeloma group reviewed myeloma patients that presented with renal impairment from 1990 to 2011 and noted that overall survival improved significantly in the last 10 years with the introduction of thalidomide in 2000 and bortezomib in 2005 (22).

HSPN has been reported very occasionally in patients with IgA monoclonal gammopathy or MM (23-25). As can 
be seen from the treatment course of our case, $\lg A$ paraproteinemia plays an important role in nephropathy. Therapeutic choices should be determined taking into account the renal characteristics of the disease, particularly the risk of renal deterioration, the presence and severity of extrarenal manifestations, and the safety profile of chemotherapy agents in renal impairment (13).

In conclusion, HSPN that cannot be relieved by treatment with glucocorticoid and immunosuppressive agents

\section{References}

1. Yang $\mathrm{YH}, \mathrm{Yu} \mathrm{HH}$, Chiang BL. The diagnosis and classification of Henoch-Schönlein purpura: an updated review. Autoimmunity Rev 2014; 13: 355-358, doi: 10.1016/j.autrev. 2014.01.031.

2. Nielsen HE. Epidemiology of Schonlein-Henoch purpura. Acta Paediatr Scand 1988; 77: 125-131, doi: 10.1111/ j.1651-2227.1988.tb10610.x.

3. Kang Y, Park JS, Ha YJ, Kang MI, Park HJ, Lee SW, et al. Differences in clinical manifestations and outcomes between adult and child patients with Henoch-Schönlein purpura. J Korean Med Sci 2014; 29: 198-203, doi: 10.3346/jkms. 2014.29.2.198.

4. Pillebout E, Thervet E, Hill G, Alberti C, Vanhille P, Nochy D. Henoch-Schönlein purpura in adults: outcome and prognostic factors. J Am Soc Nephrol 2002; 13: 1271-1278, doi: 10.1097/01.ASN.0000013883.99976.22.

5. Bridoux F, Leung N, Hutchison CA, Touchard G, Sethi S, Fermand JP, et al. Diagnosis of monoclonal gammopathy of renal significance. Kidney Int 2015; 87: 698-711, doi: 10.1038/ki.2014.408.

6. Sethi S, Fervenza FC, Rajkumar SV. Spectrum of manifestations of monoclonal gammopathy-associated renal lesions. Curr Opin Nephrol Hypertens 2016; 25: 127-137, doi: 10.1097/MNH.0000000000000201.

7. Yadav P, Leung N, Sanders PW, Cockwell P. The use of immunoglobulin light chain assays in the diagnosis of paraprotein-related kidney disease. Kidney Int 2015; 87: 692-697, doi: 10.1038/ki.2014.333.

8. Brouet JC, Clauvel JP, Danon F, Klein M, Seligmann M. Biologic and clinical significance of cryoglobulins. A report of 86 cases. Am J Med 1974; 57: 775-788, doi: 10.1016/00029343(74)90852-3.

9. Requena L. Specific cutaneous involvement in patients with multiple myeloma. A clinicopathological, immunohistochemical and cytogenetic study of 40 cases. Actas Dermosifiliogr 2005; 96: 424-440 [in Spanish], doi: 10.1016/S0001-7310 (05)73107-0.

10. Lipsker D. Monoclonal gammopathy of cutaneous significance: review of a relevant concept. J Eur Acad Dermatol Venereol 2017; 31: 45-52, doi: 10.1111/jdv.13847.

11. Mehtat Unlu S, Ozsan H, Sarioglu S. The scope of kidney affection in monoclonal gammopathies at all levels of clinical significance. Turk J Haematol 2017; 34: 282-288, doi: 10.4274/tjh.2017.0197.

12. Leung N, Bridoux F, Batuman V, Chaidos A, Cockwell $P$, D'Agati VD, et al. The evaluation of monoclonal gammopathy suggests the presence of monoclonal gammopathy in adult patients. We must bear in mind the latent presence of MGRS in cases of HSPN.

\section{Acknowledgments}

We wish to thank the patient for being willing to share his case with us. We also wish to thank Dr. Huan-ling Zhu (West China Hospital of Sichuan University) for valuable discussion.

of renal significance: a consensus report of the International Kidney and Monoclonal Gammopathy Research Group. Nat Rev Nephrol 2019; 15: 45-59, doi: 10.1038/s41581-0180077-4.

13. Correia SO, Santos S, Malheiro J, Cabrita A, Martins S, Santos J. Monoclonal gammopathy of renal significance: diagnostic workup. World J Nephrol 2017; 6: 72-78, doi: 10.5527/wjn.v6.i2.72.

14. Zand L, Nasr SH, Gertz MA, Dispenzieri A, Lacy MQ, Buadi $\mathrm{FK}$, et al. Clinical and prognostic differences among patients with light chain deposition disease, myeloma cast nephropathy and both. Leuk Lymphoma 2015; 56: 3357-3364, doi: 10.3109/10428194.2015.1040011.

15. Chauvet S, Fremeaux-Bacchi V, Petitprez F, Karras A, Daniel L, Burtey $S$, et al. Treatment of B-cell disorder improves renal outcome of patients with monoclonal gammopathy-associated C3 glomerulopathy. Blood 2017; 129: 1437-1447, doi: 10.1182/blood-2016-08-737163.

16. Leung N, Bridoux F, Hutchison CA, Nasr SH, Cockwell P, Fermand JP, et al. Monoclonal gammopathy of renal significance: when MGUS is no longer undetermined or insignificant. Blood 2012; 120: 4292-4295, doi: 10.1182/ blood-2012-07-445304.

17. Steiner N, Gobel G, Suchecki P, Prokop W, Neuwirt H, Gunsilius E. Monoclonal gammopathy of renal significance (MGRS) increases the risk for progression to multiple myeloma: an observational study of 2935 MGUS patients. Oncotarget 2017; 9: 2344-2356.

18. Vignon $M$, Javaugue $V$, Alexander MP, El-Karoui $K$, Karras A, Roos-Weil D, et al. Current anti-myeloma therapies in renal manifestations of monoclonal light chain-associated Fanconi syndrome: a retrospective series of 49 patients. Leukemia 2017; 31: 123-129, doi: 10.1038/leu. 2016.195.

19. Fermand JP, Bridoux F, Kyle RA, Kastritis E, Weiss BM, Cook MA, et al. How I treat monoclonal gammopathy of renal significance (MGRS). Blood 2013; 122: 3583-3590, doi: 10.1182/blood-2013-05-495929.

20. Tunon J, Oliva-Encabo R, Cortes M. Diagnosis of cardiac amyloidosis by skin lesions. Rev Esp Cardiol (Engl Ed) 2014; 67: 666 .

21. Manohar S, Nasr SH, Leung N. Light chain cast nephropathy: practical considerations in the management of myeloma kidney-what we know and what the future may hold. Curr Hematol Malig Rep 2018; 13: 220-226, doi: 10.1007/s11899-018-0451-0. 
22. Dimopoulos MA, Delimpasi S, Katodritou E, Vassou A, Kyrtsonis MC, Repousis P, et al. Significant improvement in the survival of patients with multiple myeloma presenting with severe renal impairment after the introduction of novel agents. Ann Oncol 2014; 25: 195-200, doi: 10.1093/annonc/mdt483.

23. Zickerman AM, Allen AC, Talwar V, Olczak SA, Brownlee A, Holland $M$, et al. IgA myeloma presenting as HenochSchönlein purpura with nephritis. Am J Kidney Dis 2000; 36: E19, doi: 10.1053/ajkd.2000.16221.
24. Dosa S, Cairns SA, Mallick NP, Lawler W, Williams G. Relapsing Henoch-Schönlein syndrome with renal involvement in a patient with an IgA monoclonal gammopathy. Nephron 1980; 26: 145-148, doi: 10.1159/000181970.

25. Meguro D, Akimoto T, Nakazawa E, Onishi A, Inoue M, Saito O, et al. Case of Henoch-Schönlein purpura nephritis associated with $\lg \mathrm{A}$ monoclonal gammopathy of undetermined significance. Nihon Zinzo Gakkai Shi 2009; 51: 145149 [in Japanese]. 